\title{
Multiple Myeloma Similar to Traumatic Trigeminal Neuropathy after Dental Treatment: Case Report
}

\author{
Ji-Yeon Lee ${ }^{1}$, Hye-Min Ju' ${ }^{1}$ Hae-Ohk Lee ${ }^{1}$, Hye-Mi Jeon ${ }^{2}$, Kyung-Hee Kim ${ }^{3}$, \\ Sung-Hee Jeong ${ }^{1}$, Yong-Woo Ahn ${ }^{1}$, Soo-Min Ok \\ 'Department of Oral Medicine, Dental Research Institute, Dental and Life Science Institute, \\ Pusan National University School of Dentistry, Yangsan, Korea \\ ${ }^{2}$ Dental Clinic Center, Pusan National University Hospital, Busan, Korea \\ ${ }^{3}$ Department of Oral Medicine, Inje University Busan Paik Hospital, Busan, Korea
}

Received June 3, 2020

Revised June 11, 2020

Accepted June 11, 2020

\section{Correspondence to:}

Soo-Min Ok

Department of Oral Medicine, School of

Dentistry, Pusan National University, 49

Busandaehak-ro, Mulgeum-eup, Yangsan

50612, Korea

Tel: +82-55-360-5243

Fax: +82-55-360-5234

E-mail:soomin0402@hanmail.net

https://orcid.org/0000-0003-1776-371X

This work was supported by a 2-year

Research Grant of Pusan National University.
Multiple myeloma is malignant neoplasm of plasma cells. Mandible lesions are rarely the first symptoms of multiple myeloma. While sensory dysfunction and pain are the main symptoms of traumatic trigeminal neuropathy, the same oral symptoms can appear in cases of multiple myeloma with developed mandible lesions. In addition, if the radiological osteoporosis or lytic lesion is seen in older patients, further examination is required to find the cause of the symptoms. In this paper, we present a case that was tentatively diagnosed as traumatic trigeminal neuropathy after left third molar extraction, but later confirmed as multiple myeloma.

Key Words: Hyperalgesia; Multiple myeloma; Trigeminal nerve injuries

\section{INTRODUCTION}

Painful post-traumatic trigeminal neuropathy (TTN) is caused by oral surgical operation, especially third molar extraction [1]. Diagnostic criteria for TTN include spontaneous or stimulus-evoked pain affecting the receptive field of one or more divisions of the trigeminal nerve, the development within three months of traumatic events, the presence of at least one neurologic dysfunction, imaging identifying a neurologic lesion, and exclusion of other diseases [2]. The clinical features of TTN can vary. Usually, burning or shooting pain occurs on the side of damaged area, and sometimes at a distance along the dermatome of the affected nerve. In addition to the pain, it is accompanied by a sensory dysfunction [3]. Sensory dysfunction can be divided into positive sign such as hyperalgesia, dysesthesia, and allodynia and negative sign such as hypoesthesia and anesthesia. Because inflammatory mediators are released from damaged areas by trauma, it mainly features such as allodynia and hyperalgesia [4].

Sensory dysfunction, one of the main diagnostic criteria for painful post-traumatic trigeminal neuropathy, can be caused by local factors such as trauma as well as systemic factors. Systemic factors are associated with systemic diseases such as multiple sclerosis, sarcoidosis, metastasis, viral and bacterial infections, leukemia, and lymphoma [5].

Among these, multiple myeloma that is common in maxillofacial area is a tumor of plasma cell origin that accounts for 1\% of all malignant tumors and 15\% of hematologic malignant tumors. It occurs mainly in elderly men.

Copyright (c) 2020 Korean Academy of Orofacial Pain and Oral Medicine. All rights reserved.

(c) This is an open-access article distributed under the terms of the Creative Commons Attribution Non-Commercial License (http://creativecommons.org/licenses/by-nc/4.0/), which permits unrestricted non-commercial use, distribution, and reproduction in any medium, provided the original work is properly cited. 
This disease is characterized by monoclonal proliferation of abnormal plasma cells. These cells produce an kappa or lambda light chain [6]. Multiple myeloma occurs in several bones, and usually appears in the jawbone in the advanced stages of the disease. The symptoms of multiple myeloma in maxillofacial lesion include odontalgia, paresthesia, gingival hemorrhage and ulcerations [7], which are easy to confuse with a dental-originated lesion. In particular, if symptoms occur after dental treatment, it may be easy to be confused with traumatic trigeminal neuropathy.

Therefore, the purpose of this study is to find out the considerations of diagnosis and treatment of multiple myeloma similar to TTN through a case report on a patient with sensory dysfunction patient and multiple myeloma-originated pain after dental treatment.

\section{CASE REPORT}

An 82-year-old female patient visited the Department of Oral Medicine at the Pusan National University Dental Hospital with the chief complaint of sensory dysfunction on the left chin and around the lip after extraction of the lower left third molar one week prior. The patient had no medical history. Clinical findings showed no signs of infection such as swelling or local fever. On panoramic view, there were no abnormal findings except the extraction socket (Fig. 1). In order to evaluate the relationship between the extraction site and the mandibular canal, a CBCT was recommended. However, the patient refused to have a CBCT taken. Based on the history and clinical findings, the tentative diagnosis was numbness, allodynia and hyperalgesia due to painful traumatic trigeminal neuropathy. Clinical mapping was performed on the sensory dysfunction area (Fig. 2A). Prednisolone (Solondo tab.; Yuhan Co., Seoul, Korea) in tapering doses (30 mg prednisolone reduced by $5 \mathrm{mg}$ daily) and gabapentin (Neurontin, $100 \mathrm{mg}$, three times a day; Pfizer, New York, NY, USA) was prescribed for 7 days, and low-level laser therapy was performed. Two days later, the patient returned to the hospital with sudden severe sharp and aching pain. Because there were no signs of infection and symptoms indicative of other disease, systemic corticosteroid (Dexamethasone disodium phosphate $5 \mathrm{mg} / \mathrm{mL}$ ) was injected directly into muscle. During the patient's third visit (five days later), the patient described the pain as seeming to decrease slightly after the injection but, that pain site was wider and more persistent. The extraction socket seemed to be in a delayed healing state with bleeding tendency on intraoral examination (Fig. 2B). Because these symptoms were different from general traumatic trigeminal neuropathy, a CBCT was again recommended to confirm the need for additional microneurosurgical treatment and to exclude the possibility of other diseases. Additional systemic steroid injection (Dexamethasone disodium phosphate $5 \mathrm{mg} / \mathrm{mL}$ ) was

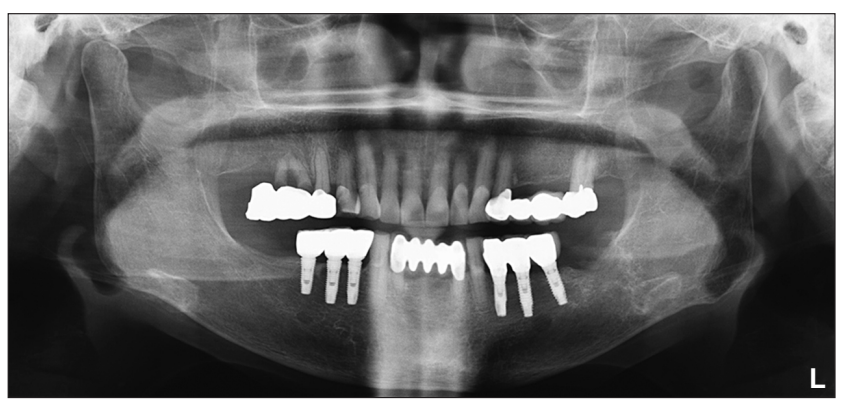

Fig. 1. Left mandible third molar extraction socket on panoramic view at first visit.
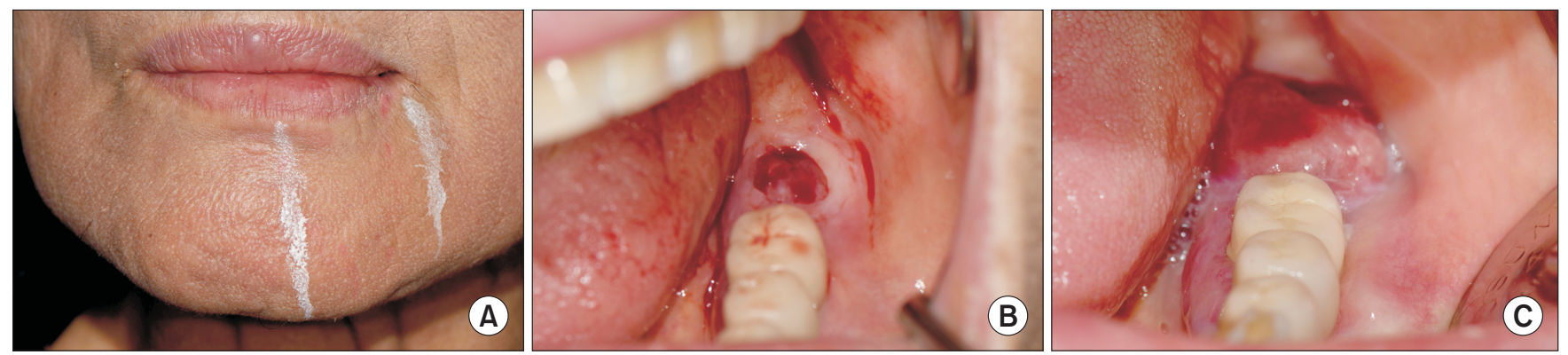

Fig. 2. (A) Clinical mapping on hypoesthesia area at first visit. (B) Delayed healing with bleeding tendency on extraction site (14 days after extraction). (C) Abnormal mass was observed in the extraction site on intraoral examination (22 days after extraction). 
performed and gabapentin (Neurontin, $100 \mathrm{mg}$, three times a day), Brufen (400 mg, three times a day; Boots Pure Drug Co., Ltd., Nottingham, UK), Mucosta (100 mg, three times a day; Otsuka Pharmaceutical Co., Ltd., Tokyo, Japan) and Augmentin (375 mg, three times a day; GlaxoSmithKline, Middlesex, UK) were prescribed for 14 days. The patient was asked to use a hexamedine gargle for 5-10 minutes three times a day. Ten days later (three weeks after extraction), the patient visited suddenly with local fever on left cheek and more pain. The CBCT taken at last visit showed osteoporosis (Fig. 3), and as a result, the cortical bone of the mandibular canal was not well observed, therefore, it was not possible to determine whether the nerve damage was caused by trauma during extraction. In addition, abnormal soft tissue mass on extraction site was observed (Fig. 2C). Based on no change of symptom in the antibiotic prescription to exclude infections, СBCT finding, and soft tissue mass, the patient was referred to the department of oral and maxillofacial surgery for biopsy. Histopathological findings resulted in plasmacytoma (Fig. 4A). The patient was referred to the department of hemato-oncology for blood test, radiologic test and bone marrow biopsy. As a result of the blood test, mild anemia, slightly decreased white blood cells, abnormal increase of Ig $\mathrm{G}$, and monoclonal peak in gamma region was observed. An increased fluorodeoxyglucose intake on positron emission tomography-computed tomography was observed in both humeri, spine, sternum, pelvic bones, femurs, left mandible, and ribs. And it was read as

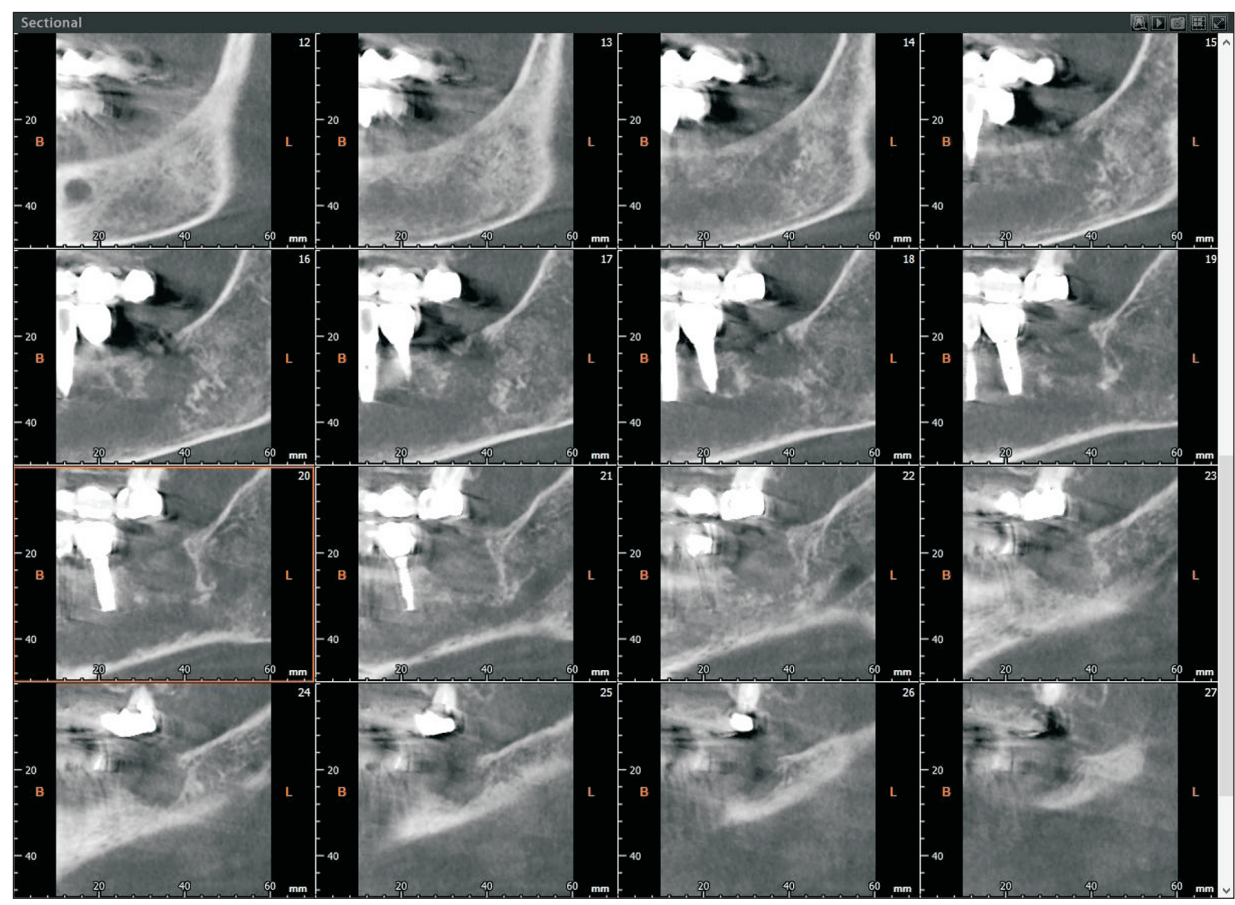

Fig. 3. Osteoporosis around the extraction socket on cone beam computed tomography view.
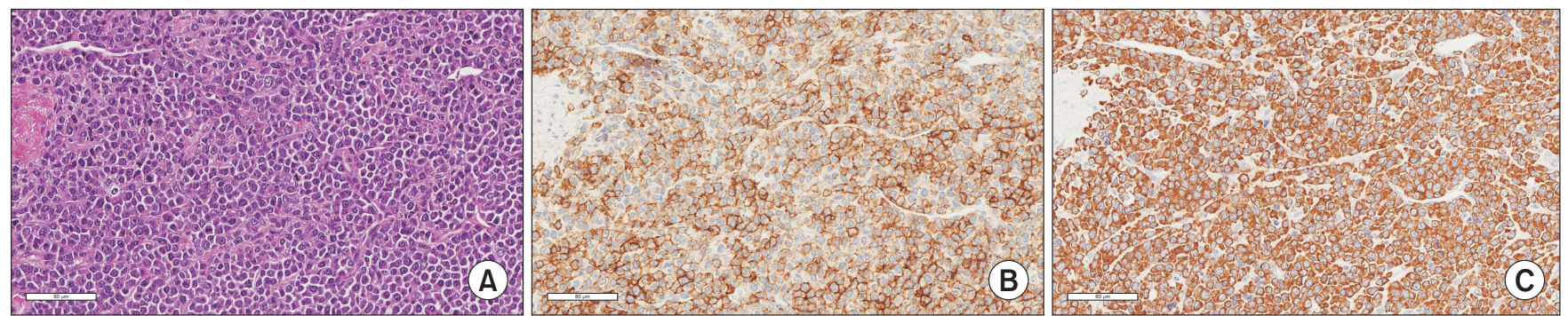

Fig. 4. Histological examination of the lesion. (A) Plasmacytoma consisting of abnormal mitosis, pleomorphism, and hyperchromatism plasma cells (H\&E staining, $\times 300$ ). (B) CD 138 positive on plasma cells (Immunohistochemistry, $\times 300$ ). (C) lambda chain positive on plasma cells (Immunohistochemistry, $\times 300)$. 
multiple bone metastasis. Abnormal plasma cell proliferation (20\%-30\%) was observed on the bone marrow biopsy, and immunohistochemistry showed positive patterns in CD138 (Fig. 4B) and lambda chains (Fig. 4C). Based on the results of these tests, multiple myeloma (Ig G, lambda restriction type) was finally diagnosed and treated.

\section{DISCUSSION}

Plasmacytoma resulting from proliferation of abnormal plasma cell is classified into multiple myeloma, solitary plasmacytoma (SBP), and extramedullary plasmacytoma (EMP). SBP and EMP are localized forms, but multiple myeloma is systemic proliferation of neoplasm cells [8].

The most common clinical features of multiple myeloma are bone pain, fatigue, and anemia. These symptoms are associated with the proliferation and expansion of abnormal plasma cells in the bone marrow. The first sign of multiple myeloma is rarely in the mandible and often occurs in advanced stage [9]. Lesions are mainly found in the posterior of mandible because of hematopoietic activity. The most common oral symptoms are swelling, bone pain, odontalgia, paresthesia, gingival hemorrhage and tooth mobility [7].

The radiological features of multiple myeloma vary. The lytic lesions appear to be multiple well defined, or irregular and diffuse radiolucent lesions [10]. In addition, it shows pathologic fractures and osteoporosis [11]. Differential diagnosis is needed for metastatic disease with bony destruction, osteomyelitis, and benign odontogenic tumors [10].

In this case, the patient presented hypoesthesia, allodynia and hyperalgesia with sharp and aching pain 1 week after extraction. The patient had no medical history. No abnormality was found in the intraoral examination and panoramic view. Based on persistent pain after dental treatment and sensory dysfunction such as paresthesia and hypoesthesia, the tentative diagnosis was painful traumatic trigeminal neuropathy. In order to identify damage to mandibular canal and causes of pain other than nerve damage, CBCT taking was planned, but delayed due to patient's rejection. At second visit, because of severe pain without infection symptom such as swelling and local fever, systemic corticosteroid injection was applied. In general, posttraumatic neuropathy due to unidentified inflammation has progressive symptoms, severe pain and it has been treated with analgesic, but recently corticosteroid therapy is performed to reduce the duration of pain [12]. However, the patient had little response to anti-inflammatory analgesics and high-dose steroid therapy with persistent severe pain. To clearly identify the cause of pain, a CBCT was again recommended and taken with the patient's consent. Because no clear radiolucent lesion was observed on $\mathrm{CBCT}$, bone lesion was excluded. In addition, because of osteoporosis, the cortical bone of the mandibular canal was not well observed, so it was difficult to diagnose damage to the mandibular canal by trauma. However, additionally the mass of extraction site occurred and was diagnosed as plasmacytoma by biopsy. The patient was referred to hemato-oncology and the final diagnosis was multiple myeloma.

There were no specific underlying diseases in history taking and apparent abnormal lesions on radiographic findings at first visit, but sensory dysfunction with severe bone pain in the oral cavity was the first sign of multiple myeloma. The patient did not complain of any systemic symptoms but anemia was observed on the blood test. This is a symptom related to prognosis (CRAB; hypercalcemia, renal deficiency, anemia, bone lesions), which are typical clinical manifestations for multiple myeloma [13]. Multiple myeloma should be diagnosed as plasmacytoma with a bone biopsy, or clonal plasma cells of bone marrow should be more than 10\% [14]. In this case, bone marrow biopsy showed nearly 70\% hypercellular marrow with proliferation plasma cells $(20 \%-$ 30\%). Immunohistochemical analysis revealed monoclonal Ig G staining with lambda chain.

In conclusion, even in the absence of a medical history, if older patients complain of severe pain and sensory dysfunction, and osteoporosis or lytic lesion are observed on the radiographs, the dentist must make additional screening test decisions, such as blood tests for excluding the association with systemic disease, through frequent re-call checks. Early diagnosis of multiple myeloma affects survival rates, so it is important for dentists to recognize possible symptoms in the oral cavity such as swelling and osteolytic lesion and refer for accurate diagnosis and treatment [15]. 


\section{CONFLICT OF INTEREST}

No potential conflict of interest relevant to this article was reported.

\section{ORCID}

\author{
Ji-Yeon Lee \\ https://orcid.org/0000-0001-9654-5723 \\ Hye-Min Ju \\ https://orcid.org/0000-0002-9252-6717 \\ Hae-Ohk Lee \\ https://orcid.org/0000-0002-9859-5342 \\ Hye-Mi Jeon \\ https://orcid.org/0000-0003-0007-5662 \\ Kyung-Hee Kim \\ https://orcid.org/0000-0003-2922-6452 \\ Sung-Hee Jeong \\ https://orcid.org/0000-0002-6296-4775 \\ Yong-Woo Ahn \\ https://orcid.org/0000-0002-2197-0394 \\ Soo-Min Ok \\ https://orcid.org/0000-0003-1776-371X
}

\section{REFERENCES}

1. Peñarrocha MA, Peñarrocha D, Bagán JV, Peñarrocha M. Posttraumatic trigeminal neuropathy. A study of 63 cases. Med Oral Patol Oral Cir Bucal 2012;17:e297-e300.

2. Benoliel R, Zadik Y, Eliav E, Sharav Y. Peripheral painful traumatic trigeminal neuropathy: clinical features in 91 cases and proposal of novel diagnostic criteria. J Orofac Pain 2012;26:4958.

3. Benoliel R, Teich S, Eliav E. Painful traumatic trigeminal neuropathy. Oral Maxillofac Surg Clin North Am 2016;28:371-380.

4. Benoliel R, Kahn J, Eliav E. Peripheral painful traumatic trigeminal neuropathies. Oral Dis 2012;18:317-332.

5. Mohammadi Z. Endodontics-related paresthesia of the mental and inferior alveolar nerves: an updated review. J Can Dent Assoc 2010;76:a117.

6. Sreeja C, Vijayabanu B, Vijayalakshmi D, Devi M, Ramakrishnan K, Dhivya K. Multiple myeloma involving mandible: in an elderly female. J Pharm Bioallied Sci 2015;7(Suppl 2):S763-S765.

7. Ali IK, Parate AR, Kasat VO, Dora A. Multiple myeloma with primary manifestation in the mandible. Cureus 2018;10:e2265.

8. Dayisoylu EH, Ceneli 0, Coskunoglu EZ. Solitary plasmacytoma of the mandible: an uncommon entity. Iran Red Crescent Med J 2016;18:e22932.

9. Ramaiah KK, Joshi V, Thayi SR, Sathyanarayana P, Patil P, Ahmed Z. Multiple myeloma presenting with a maxillary lesion as the first sign. Imaging Sci Dent 2015;45:55-60.

10. Fregnani ER, Leite AA, Parahyba CJ, Nesrallah AC, Ramos-Perez FM, Perez DC. Mandibular destructive radiolucent lesion: the first sign of multiple myeloma. J Clin Exp Dent 2016;8:e465-e468.

11. Kyle RA, Gertz MA, Witzig TE, et al. Review of 1027 patients with newly diagnosed multiple myeloma. Mayo Clin Proc 2003;78:2133.

12. Silbert BI, Kolm S, Silbert PL. Postprocedural inflammatory inferior alveolar neuropathy: an important differential diagnosis. Oral Surg Oral Med Oral Pathol Oral Radiol 2013;115:e1-e3.

13. Talamo G, Farooq U, Zangari M, et al. Beyond the CRAB symptoms: a study of presenting clinical manifestations of multiple myeloma. Clin Lymphoma Myeloma Leuk 2010;10:464-468.

14. Fazel F, Bassa F. An approach to the diagnosis and management of multiple myeloma. S Afr Med J 2019;109:723-727.

15. Almeida TMX, Cavalcanti ÉFF, Freitas ADS, Magalhães RJP, Maiolino A, Torres SR. Can dentists detect multiple myeloma through oral manifestations? Rev Bras Hematol Hemoter 2018; 40:43-49. 